\title{
Recent Developments in the Management of Advanced Prostate Cancer
}

\author{
Presented by Sandy Srinivas, MD
}

\section{ABSTRACT}

Numerous new options are available for the treatment of prostate cancer when it progresses beyond localized disease. Older drugs, first developed for treatment of metastatic castration-resistant prostate cancer (CRPC), are being used to treat nonmetastatic disease based on results of large randomized controlled trials. Sequencing of available treatment options is challenging for CRPC, but there is some guidance from trial data. Cross-resistance among newer hormonal drugs is a concern, and therefore switching to another class of drug is preferred. Emerging treatments on the horizon, such as PARP inhibitors in patients with BRCA2 mutations and lutetium-177 in those with prostate-specific membrane antigen (PSMA)-positive disease, may look promising to improve outcomes among those with advanced prostate cancer.

J Natl Compr Canc Netw 2020;18(7.5):969-972 doi: 10.6004/jnccn.2020.5021

Prostate cancer can evolve from clinically localized disease to biochemically resistant disease (signaled by an increase in prostate-specific antigen [PSA]). The disease can then progress to nonmetastatic castration-sensitive prostate cancer (CSPC), nonmetastatic castration-resistant prostate cancer (CRPC), and eventually metastatic CRPC, which is a lethal phenotype, explained Sandy Srinivas, MD, Professor, Stanford Cancer Institute. At the NCCN 2020 Virtual Annual Conference, Dr. Srinivas outlined current NCCN Guidelines recommendations for the treatment of advanced prostate cancer.

"Traditionally, the drugs used to treat progressive prostate cancer, such as androgen deprivation therapy [ADT], have not improved overall survival [OS]. Many drugs used in clinical practice had not met the endpoint of OS," she said.

\section{Nonmetastatic CRPC}

Nonmetastatic CSPC is characterized by biochemical recurrence and treated with ADT. However, despite low levels of testosterone, PSA levels will increase, and these patients are then categorized as nonmetastatic CRPC. "A large number of these patients have no evidence of disease on nuclear scans; CT imaging of the chest, abdomen, and pelvis; or MRI, and become castration-resistant," she said.

Three randomized trials in nonmetastatic CRPC had a similar design and used a unique endpoint of metastasis-free survival to study novel androgen receptor (AR)-directed hormonal agents plus ADT versus ADT alone: SPARTAN (apalutamide), ${ }^{1}$ PROSPER (enzalutamide), ${ }^{2}$ and ARAMIS (darolutamide). ${ }^{3}$ All patients enrolled in these trials had a PSA doubling time (PSADT) of $\leq 10$ months and no evidence of metastatic disease on conventiona imaging. "The 3 drugs [in these trials] have a similar mechanism of action but had different side-effect profiles," Dr. Srinivas said.

"The most important side effects of these drugs are fatigue, falls, and fractures." However, the side-effect profiles of the drugs are somewhat different. The risk of fatigue is higher for apalutamide and enzalutamide (27\%-30\%) compared with darolutamide (12\%). Falls were reported in $12 \%$ to $14 \%$ of patients treated with apalutamide and enzalutamide versus $4.2 \%$ of those treated with darolutamide. Fractures were reported in $12 \%$ of apalutamide-treated patients versus $4.2 \%$ of those treated with darolutamide. ${ }^{1-3}$ Rash is unique to apalutamide.

"In these trials, more than two-thirds of patients had a PSADT $<6$ months," she noted (Figure 1), and $90 \%$ of patients were not treated with a bone-targeted agent, such as denosumab or zoledronic acid.

All 3 trials met the primary endpoint of metastasisfree survival with novel AR-directed therapies (Figure 2); OS data are not yet mature. Median metastasis-free survival was 36.6 months for enzalutamide versus 14.7 months for placebo in PROSPER, 40.5 months for apalutamide versus 16.2 months for placebo in SPARTAN, and 40.4 months for darolutamide versus 18.4 months for placebo in ARAMIS. ${ }^{1-3}$ "These benefits [of these drugs] come with a price to pay, given that these patients are completely asymptomatic and have no evidence of metastatic disease," Dr. Srinivas pointed out.

Level 1 evidence from these phase III trials has led to the current recommendations in the NCCN Clinical Practice Guidelines for Oncology (NCCN Guidelines) for 


\begin{tabular}{|c|c|c|c|c|c|c|}
\hline \multirow[b]{2}{*}{ Characteristic } & \multicolumn{2}{|c|}{ PROSPER $^{2}$} & \multicolumn{2}{|c|}{ SPARTAN $^{1}$} & \multicolumn{2}{|c|}{ ARAMIS $^{3}$} \\
\hline & $\begin{array}{c}\text { Enzalutamide + } \\
\text { ADT } \\
(n=933)\end{array}$ & $\begin{array}{c}\text { Placebo + ADT } \\
(n=468)\end{array}$ & $\begin{array}{c}\text { Apalutamide + } \\
\text { ADT } \\
(n=806)\end{array}$ & $\begin{array}{c}\text { Placebo + ADT } \\
(n=401)\end{array}$ & $\begin{array}{c}\text { Darolutamide + ADT } \\
(n=955)\end{array}$ & $\begin{array}{l}\text { Placebo + ADT } \\
(n=554)\end{array}$ \\
\hline Median age, y & 74 & 73 & 74 & 74 & 74 & 74 \\
\hline Median PSADT, mo & 3.8 & 3.6 & 4.4 & 4.5 & 4.4 & 4.7 \\
\hline \multicolumn{7}{|l|}{ PSADT, $\%$} \\
\hline$<6 \mathrm{mo}$ & 77 & 77 & 72 & 71 & 70 & 67 \\
\hline$\geq 6 \mathrm{mo}$ & 23 & 23 & 29 & 29 & 30 & 33 \\
\hline \multicolumn{7}{|c|}{ Baseline bone-targeted agent, $\%$} \\
\hline No & 89 & 90 & 90 & 90 & 97 & 94 \\
\hline Yes & 11 & 10 & 10 & 10 & 3 & 6 \\
\hline
\end{tabular}

Figure 1. PROSPER/SPARTAN/ARAMIS demographics.

Abbreviations: ADT, androgen deprivation therapy; PSADT, prostate-specific antigen doubling time.

Prostate Cancer, version 1.2020. Recommendations for nonmetastatic CRPC are divided into 2 groups: PSADT $>10$ months and PSADT $\leq 10$ months. Patients with PSADT $>10$ months are managed with observation or secondary hormonal therapy, whereas those with PSADT $\leq 10$ months can be treated with any of the 3 agents (all category 1 recommendations): apalutamide, enzalutamide, and darolutamide.

More sensitive imaging tests in development reveal the presence of metastases in patients with nonmetastatic CRPC that are not visible on conventional imaging with CT and MRI. "Using these newer tests, such as prostate-specific membrane antigen [PSMA] PET, may eliminate this disease state," she said.

Furthermore, Dr. Srinivas cautions that "these 3 novel AR-directed hormonal agents are cross-resistant. All 3 therapies are appropriate treatment for patients with nonmetastatic CRPC and short PSADT."

\section{Metastatic Prostate Cancer}

Results of recent trials in metastatic prostate cancer have led to a number of changes in the NCCN Guidelines. For patients with metastatic castrationnaïve disease, 4 drugs have category 1 recommendations (apalutamide, abiraterone acetate, docetaxel, and enzalutamide) and fine-particle abiraterone is a category $2 \mathrm{~B}$ recommendation (all given with an $\mathrm{ADT}$ backbone). For low-volume metastatic disease, ADT with external-beam radiation therapy to the primary tumor or ADT alone is recommended. Support for the use of docetaxel in patients with castration-naïve prostate cancer was based on results of 3 large, randomized, phase III trials: GETUG-15, ${ }^{4}$ CHAARTED,${ }^{5}$ and STAMPEDE. ${ }^{6}$ In these studies, patients received 6 to 9 cycles of docetaxel + ADT versus ADT alone.
GETUG-15 enrolled 385 patients, CHAARTED enrolled 790, and STAMPEDE enrolled 2,962. All 3 trials met the primary endpoint of progression-free survival (PFS) with the addition of docetaxel. - $^{4-6}$ "Both CHAARTED and STAMPEDE showed that adding docetaxel to ADT improved OS by 10 to 13 months; this changed practice for adopting docetaxel for metastatic castration-naïve disease," Dr. Srinivas said. Further analysis of the CHAARTED trial results suggested that the magnitude of benefit was much greater in patients with high-volume disease; those with low-volume disease $(<4$ metastases $)$ did not experience a benefit. ${ }^{7}$

Four large trials support the use of AR inhibitors in castration-naïve prostate cancer: LATITUDE (abiraterone acetate), ${ }^{8}$ ARCHES (enzalutamide), ${ }^{9}$ TITAN (apalutamide), ${ }^{10}$ and ENZAMET (enzalutamide). ${ }^{11}$ More than 1,000 patients were enrolled in each of these trials, and the median age was approximately 69 to 70 years. One difference among these trials was the volume of disease in the patient population; other differences included the percentage of patients previously treated with docetaxel. LATITUDE was designed before docetaxel was used to treat metastatic CSPC, thus no patients received docetaxel. Conversely, almost $20 \%$ of patients enrolled in ARCHES and TITAN had received prior docetaxel, whereas $45 \%$ had in ENZAMET.

LATITUDE was designed as a trial for patients with high-volume disease, $98 \%$ of whom met the criteria. ARCHES, TITAN, and ENZAMET had a decreased percentage of patients with high-volume disease, ranging from $52 \%$ to $62 \%$. "Disease volume has become an important criterion" for selection of therapy in metastatic CSPC, Dr. Srinivas emphasized.

All 4 trials showed significant improvement in radiographic PFS with the addition of an AR-targeted novel hormonal therapy to ADT. LATITUDE, the most mature 


\begin{tabular}{|c|c|c|c|c|c|c|}
\hline \multirow[b]{2}{*}{ Characteristic } & \multicolumn{2}{|c|}{$\mathrm{PROSPER}^{2}$} & \multicolumn{2}{|c|}{ SPARTAN $^{1}$} & \multicolumn{2}{|c|}{ ARAMIS $^{3}$} \\
\hline & $\begin{array}{c}\text { Enzalutamide + } \\
\text { ADT } \\
(n=933)\end{array}$ & $\begin{array}{c}\text { Placebo + ADT } \\
(n=468)\end{array}$ & $\begin{array}{c}\text { Apalutamide + ADT } \\
(n=806)\end{array}$ & $\begin{array}{c}\text { Placebo + ADT } \\
(n=401)\end{array}$ & $\begin{array}{c}\text { Darolutamide + ADT } \\
(n=955)\end{array}$ & $\begin{array}{l}\text { Placebo + ADT } \\
(n=554)\end{array}$ \\
\hline MFS, mo & 36.6 & $14.7 ; \mathrm{HR}, 0.29$ & 40.5 & 16.2; HR, 0.28 & 40.4 & $18.4 ; H R, 0.41$ \\
\hline $\mathrm{OS}$, mo & NR & NR & NR & $39 ; \mathrm{HR}, 0.70$ & NR & $N R ; H R, 0.71$ \\
\hline PSA progression & 37.2 & 3.9; HR, 0.07 & NR & 3.7; HR, 0.06 & 33.2 & 7.3; HR, 0.13 \\
\hline Fatigue & 27 & 3 & 30 & 21 & 12.1 & 8.7 \\
\hline Falls (\%) & 12 & 3 & 16 & 0.8 & 4.2 & 4.7 \\
\hline Fractures (\%) & & & 12 & 6.5 & 4.2 & 3.6 \\
\hline
\end{tabular}

Figure 2. PROSPER/SPARTAN/ARAMIS results.

Abbreviations: ADT, androgen deprivation therapy; HR, hazard ratio; MFS, metastasis-free survival; NR, not reported; OS, overall survival; PSA, prostate-specific antigen.

trial, showed improvement in OS. ${ }^{8-11}$ "An important lesson from these trials is that AR-directed drugs should not be added to docetaxel. When docetaxel is not added, OS is improved," she said.

The ARASENS trial is comparing darolutamide + $\mathrm{ADT}+$ docetaxel versus ADT + docetaxel alone in metastatic castration-naïve prostate cancer, and it is anticipated that results will shed more light on the effect of adding all existing effective drugs.

"We have an abundance of options for first-line castration-naïve prostate cancer, which include docetaxel, abiraterone, or enzalutamide. The choice of one drug over another depends on patient preference for intravenous [with docetaxel] or oral delivery and duration of treatment. Docetaxel is given for 18 weeks, whereas the other drugs are given longer-term until disease progression. Also, steroids are needed with both docetaxel and abiraterone acetate," she elaborated. Docetaxel is preferred for high-volume disease, whereas abiraterone acetate, enzalutamide, and apalutamide can be used for both high- and low-volume disease.

Adverse event profiles differ among these drugs. Adverse events associated with docetaxel include fatigue and neuropathy; for abiraterone acetate, they include hypertension and liver enzyme abnormalities; and for enzalutamide and apalutamide, they include falls, fracture, and rash. Patient costs and copayments are also important considerations in the choice of oral agents.

"It's good that we have all those options for castrationnaïve prostate cancer. In my practice, I treat according to disease volume. Patients with high-volume disease can receive docetaxel, enzalutamide, abiraterone acetate, or apalutamide. Low-volume disease is treated with ADT, enzalutamide, abiraterone acetate, or apalutamide," she told listeners.

\section{Metastatic CRPC}

Multiple classes of agents are approved for the treatment of metastatic CRPC, including hormonal agents (abiraterone acetate, enzalutamide), immunotherapies (sipuleucel-T), pembrolizumab for microsatellite instability-high tumors, cytotoxic agents (docetaxel, cabazitaxel), and agents that cause DNA damage (radium-223).

"We have a shuffling of the deck, because many of these drugs are being used in earlier disease states. Sipuleucel-T is used for both asymptomatic patients and post-docetaxel. Pembrolizumab is indicated across all microsatellite instability-high cancers. Bone-modifying agents [denosumab and zoledronic acid] are also used to treat metastatic CRPC, all against the backbone of ADT throughout the course of disease," she said. The 2020 NCCN Guidelines incorporate all of these drugs. For firstline therapy, abiraterone acetate, enzalutamide, docetaxel, sipuleucel-T, and radium-223 (for bone metastasis) are all category 1 recommendations. Mitoxantrone is recommended for palliation in symptomatic patients with visceral metastasis who are unable to tolerate other therapies. Other recommended therapies include fine-particle abiraterone acetate and secondary hormonal therapy.

The choice of second-line therapy depends on which drug the patient was given as first-line therapy. If firstline therapy was abiraterone acetate or enzalutamide, then docetaxel or sipuleucel-T is preferred. If docetaxel was given in the first-line, then abiraterone acetate, enzalutamide, or cabazitaxel can be given. "Sequencing of these drugs is unclear and depends on first-line treatment. Remember, there is significant cross-resistance among these drugs. Incorporating another drug with a different mechanism of action buys more time and gets the most out of these drugs in sequence," Dr. Srinivas said.

Recently, the randomized CARD trial addressed sequencing in metastatic CRPC. ${ }^{12}$ Patients whose disease progressed on abiraterone acetate or enzalutamide before or after docetaxel were randomized to cabazitaxel versus switching to another AR-targeted agent, dependant on first-line therapy. This trial demonstrated the superiority of cabazitaxel over a second AR-directed drug 
as third-line therapy for all endpoints, including PFS and OS. "In my practice, I try not to give 2 AR-directed drugs back-to-back. I incorporate radium-223 for bone metastasis or docetaxel to be sandwiched between the 2 drugs," Dr. Srinivas said.

\section{On the Horizon}

Promising emerging approaches included PARP inhibitors in selected patients with BRCA2 mutations and lutetium-177 (Lu-177), a PSMA-directed therapy.

The PROfound trial compared the PARP inhibitor olaparib versus physician's choice in patients with metastatic CRPC who progressed on prior abiraterone or enzalutamide and had a BRCA1, BRCA2, or APN mutation in cohort 1 ; cohort 2 included all other genetic alterations. ${ }^{13}$

Results showed that olaparib had the highest impact in patients with BRCA2 mutations and improved radiographic PFS by approximately 11 months; however, much less benefit was seen in all other patients.

Lu-177 given for 1 to 4 cycles was found to improve outcomes in a small study of heavily pretreated patients with PSMA-positive metastatic CRPC. ${ }^{14}$ At 12 weeks, a $\geq 50 \%$ PSA response was observed in a large number of patients, with long-lasting responses. Based on these promising results, the ongoing phase III randomized VISION trial was initiated in metastatic CRPC (ClinicalTrials.gov identifier: NCT03511664). Patients must be PSMA-positive on PET scan and have undergone prior treatment with a taxane and a novel AR-directed therapy. Patients are randomized 2:1 to Lu-177 plus best supportive care versus best supportive care alone. Endpoints are radiographic PFS and OS. The trial has completed enrollment and results are eagerly awaited by the prostate cancer community, Dr. Srinivas commented.

"I'm excited that emerging therapies with PARP inhibitors and PSMA-targeted drugs are awaited and will hopefully improve outcomes in metastatic CRPC," she said.

Disclosures: Dr. Srinivas has disclosed that she receives grant/research support from Bayer Healthcare, Endocyte, Exelixis Inc., and Genentech, Inc., and that she is a scientific advisor for Bayer Healthcare.

Correspondence: Sandy Srinivas, MD, Stanford Cancer Institute, 875 Blake Wilbur Drive, Stanford, CA 94305. Email: sandysri@stanford.edu

\section{References}

1. Smith MR, Saad F, Chowdhury S, et al. Apalutamide treatment and metastasis-free survival in prostate cancer. N Engl J Med 2018;378 1408-1418.

2. Hussain M, Fizazi K, Saad F, et al. Enzalutamide in men with nonmetastatic castration-resistant prostate cancer. N Engl J Med 2018;378:2465-2474.

3. Fizazi $\mathrm{K}$, Shore N, Tammela TL, et al. Darolutamide in nonmetastaticcastration-resistant prostate cancer. N Engl J Med 2019;380:1235-1246.

4. Sweeney CJ, Chen YH, Carducci M, et al. Chemohormonal therapy in metastatic hormone-sensitive prostate cancer. N Engl J Med 2015;373: 737-746.

5. Gravis G, Fizazi K, Oudard S, et al. Androgen-deprivation therapy alone or with docetaxel in non-castrate metastatic prostate cancer: a randomized, open-label, phase 3 trial. Lancet Oncol 2013;14:149-158.

6. James ND, Sydes MR, Clarke NW, et al. Addition of docetaxel, zoledronic acid, or both to first-line long-term hormone therapy in prostate cancer (STAMPEDE): survival results from an adaptive, multiarm, multistage, platform randomized controlled trial. Lancet 2016;387:1163-1177.

7. Kyriakopoulos CE, Chen YH, Carducci MA, et al. Chemohormonal therapy in metastatic hormone-sensitive prostate cancer: long-term survival analysis of the randomized phase III E3805 CHAARTED trial. J Clin Oncol 2018;36:1080-1087

8. Fizazi K, Tran N, Fein L, et al. Abiraterone acetate plus prednisone in patients with newly diagnosed high-risk metastatic castration-sensitive prostate cancer (LATITUDE) final overall analysis of a randomized, doubleblind, phase 3 trial. Lancet Oncol 2019;20:686-700.

9. Armstrong A, Szmulewitz RZ, Petrylak D, et al. ARCHES: a randomized, phase III study of androgen deprivation therapy with enzalutamide or placebo in met with metastatic hormone-sensitive prostate cancer. J Clin Oncol 2019;37:2974-2986.

10. Chi KN, Agarwal N, Bjartell A, et al. Apalutamide for metastatic, castration-sensitive prostate cancer. N Engl J Med 2019;381:13-24.

11. Davis ID, Martin AJ, Stockler MR, et al. Enzalutamide with standard firstline therapy in metastatic prostate cancer. N Engl J Med 2019;381: 121-131.

12. DeWit $\mathrm{R}$, de Bono J, Sternberg $\mathrm{CN}$, et al. Cabazitaxel versus abiraterone or enzalutamide in metastatic prostate cancer. N Engl J Med 2019;38: 2506-2518.

13. Hussain M, Mateo J, Fizazi K, et al. PROfound: phase 3 study of olaparib vs. enzalutamide or abiraterone for metastatic castrationresistant prostate cancer with homologous recombination repair gene alterations [abstract]. Ann Oncol 2019;30(Suppl 5):Abstract LBA_12PR.

14. Hofman MS, Violet J, Hicks RJ, et al. [177Lu]-PSMA-617 radionuclide treatment in patients with metastatic castration-resistant prostate cancer (LuPSMA trial): a single-center, single-arm, phase 2 study. Lancet Oncol $2018 ; 19: 825-833$ 